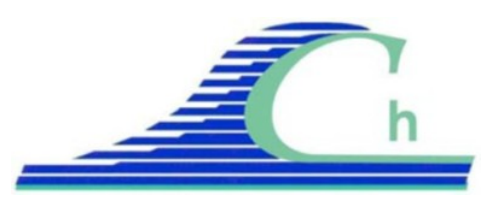

XII ${ }^{\text {emes }}$ Journées Nationales Génie Côtier-Génie Civil

Cherbourg, $12-14$ juin 2012

DOI:10.5150/jngcgc.2012.013-S C Editions Paralia CFL

disponible en ligne - http://www.paralia.fr - available online

\title{
Distributions verticales d'asymétrie et de skewness dans une couche limite sur fond mobile : comparaison expériences - modèle $k-\omega$
}

\author{
Léandro SUAREZ ${ }^{1,2}$, Eric BARTHELEMY ${ }^{2}$, \\ Céline BERNI ${ }^{2}$, Julien CHAUCHAT ${ }^{2}$
}

1. Departamento de Ingeniería Hidráulica y Ambiental, Pontificia Universidad Católica de Chile, Vicuña Mackenna 4860, casilla 306, correo 221, Santiago de Chile, Chile. lsuarez2@uc.cl

2. Laboratoire des Ecoulements Géophysiques et Industriels (UJF, GINP, CNRS), BP53, 38041 Grenoble Cedex 9, France eric.barthelemy@legi.grenoble-inp.fr ; celine.berni@legi.grenoble-inp.fr ; julien.chauchat@legi.grenoble-inp.fr

\section{Résumé :}

Lorsque les vagues se rapprochent du rivage, leurs non linéarités augmentent, et prennent une importance considérable dans l'interaction entre les vagues et le fond. Ces interactions se produisent principalement dans la couche limite turbulente.

Des expériences sur un modèle physique de vagues non linéaires sur fond mobile (BERNI, 2011), nous permettent d'obtenir les profils de vitesse ainsi que l'évolution du fond de manière couplée. Les données expérimentales obtenues suggèrent une transformation au sein de la couche limite, le skewness adimensionnel augmentant et l'asymétrie adimensionnelle diminuant au fur et à mesure que l'on se rapproche du fond. Par ailleurs on constate (DICK \& SLEATH, 1991) que l'échelle verticale de diffusion de cette couche limite est plus importante sur fond mobile que sur fond fixe.

Notre objectif est de reproduire ces 2 aspects à partir d'un modèle de couche limite turbulente de type $k$ - $\omega$ (WILCOX, 2006 ; GUIZIEN et al., 2003) et de pouvoir obtenir une meilleure description sur l'évolution des non-linéarités des vagues au sein de la couche limite. Le modèle numérique utilisé est capable de calculer la couche limite turbulente sur un fond fixe. En modélisant les mouvements du fond et en les couplant avec l'évolution de la couche limite, nous reproduisons les principales caractéristiques de l'évolution de la couche limite turbulente sur un fond mobile.

\section{Mots-clés :}

Couche limite turbulente - Non-linéarité - Fond mobile - Modèle numérique $k$ - $\omega$

\section{Abstract:}

As the waves approach the coast, non-linearities become increasingly important. The interactions between the waves and the bottom occur within the turbulent boundary layer, which is why its study represents a mean to understand the evolution of these non-linearities near the coast. 
Experimental measurements (BERNI, 2011), concerning non-linear waves on a mobile bed, provide velocity profiles and bed position in a coupled way. These data suggest a transformation within the boundary layer, with a non-dimensional skewness increasing and a non-dimensional asymmetry diminishing as we approach the bottom.

We intend to reproduce this phenomenon with a $k$ - $\omega$ numerical model (WILCOX, 2006; GUIZIEN et al., 2003), and get a better resolution on the non-linearities evolution inside the turbulent boundary layer. The numerical model is able to determine the velocity within the boundary layer on a fixed bed, by modeling the bed mobility and coupling it with the $k$ - $\omega$ model, we are able to reproduce the experimental results observed, which would indicate that the bed mobility is responsible for a vertical diffusion within the boundary layer.

\section{Keywords:}

Turbulent boundary layer - Non linearity - Mobile bed $-k-\omega$ numerical model

\section{Introduction}

La couche limite turbulente sous les vagues est sujette à différents processus, et représente une zone importante pour déterminer la contrainte de cisaillement au fond ainsi que les processus de transport de sédiment. A l'approche de la côte, la levée des vagues produit des non-linéarités, qui, influent sur la contrainte de cisaillement sur le fond. La connaissance de cette contrainte est utile pour permettre d'estimer le transport sédimentaire, d'autant plus que celui-ci s'effectue sur fond mobile.

Dans cet article, nous nous limiterons à l'étude de la couche limite turbulente sous des vagues non-linéaires et sur un fond mobile. De récentes expériences sur fond mobile (BERNI, 2011) ont permis de dégager d'intéressantes propriétés concernant les nonlinéarités dans la couche limite turbulente. Le but de cet article est de reproduire les comportements observés à l'aide d'un modèle numérique 1D de type $k$ - $\omega$ (WILCOX, 2006 ; GUIZIEN et al., 2003) qui donne des résultats sur fond fixe.

\section{Matériel et méthodes}

\subsection{Expérience}

L'expérience s'est déroulée dans le canal à houle du Laboratoire des Écoulements Géophysiques et Industriels (LEGI) dont le schéma se trouve figure 1. Ce canal mesure $36 \mathrm{~m}$ de long, $55 \mathrm{~cm}$ de large et 1,30 m de haut, et ses parois latérales sont constituées de verre. Le sédiment présent dans le fond du canal est en matière plastique (PMMA) de faible masse volumique $\left(\rho_{s}=1190 \mathrm{~g} \mathrm{~L}^{-1}\right)$, ce qui permet d'assurer une similitude des nombres de Froude et de Shields. 


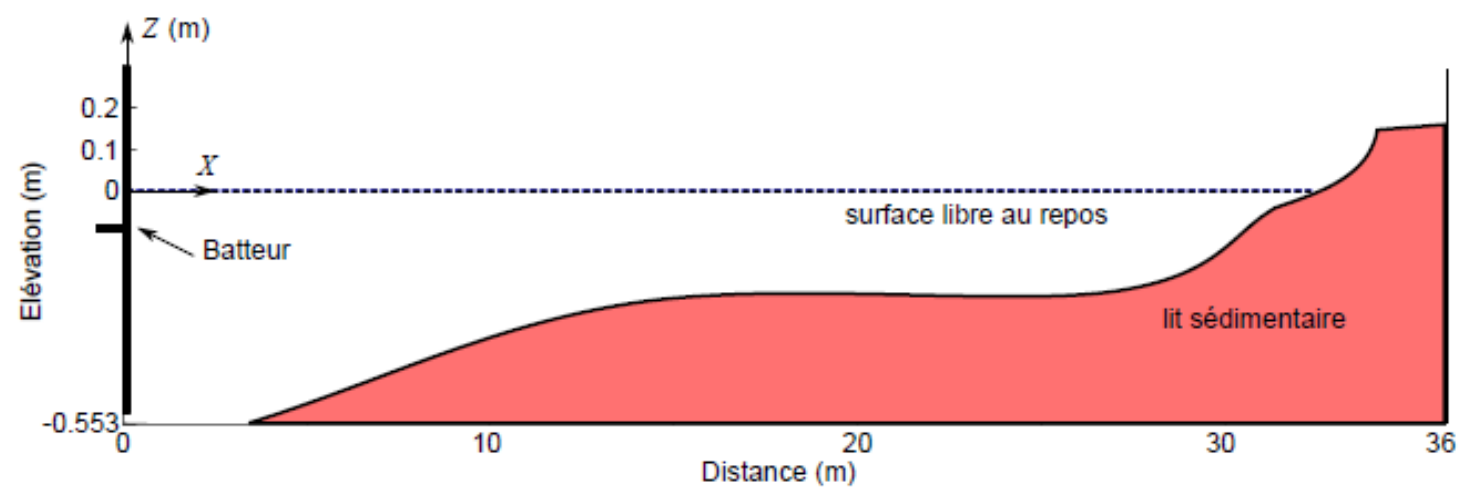

Figure 1. Schéma du canal à houle du LEGI. Un batteur piston en $x=0$ engendre deux paquets d'ondes, bichromatiques.

La houle utilisée pour l'expérience est de type bichromatique, i.e. la somme de deux ondes sinusoïdales de fréquence proche et d'amplitude égale, afin d'obtenir une onde de haute fréquence de période $T=2,5 \mathrm{~s}$ modulée par une onde de basse fréquence (figure 2). Le forçage de l'expérience consiste en une série de 23 paquets d'onde, et nous nous focalisons sur une séquence de 10 secondes dans chaque paquet, correspondant à 4 vagues passant au droit des capteurs, comme indiqué sur la figure 2.

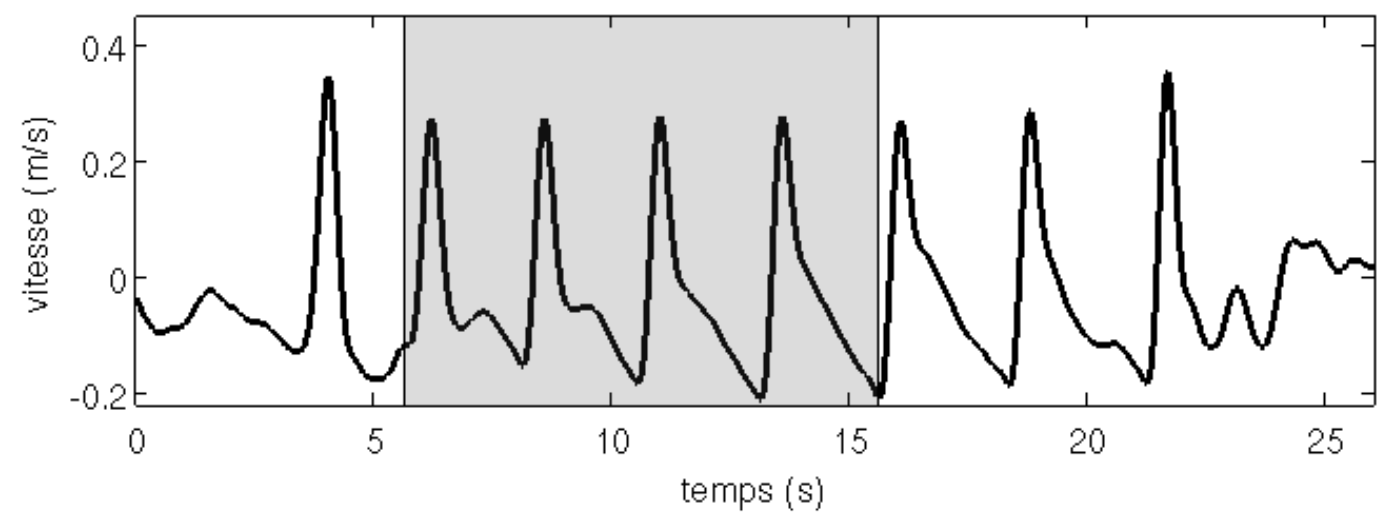

Figure 2. Vitesse moyenne sur les 23 réalisations du paquet d'ondes envoyé à chaque réalisation. En grisé, les 10 secondes prises en compte dans chaque réalisation.

A l'aide d'un profileur acoustique ADVP, BERNI (2011) obtient la position du fond, ainsi que le profil de vitesse, avec une résolution spatiale verticale de l'ordre de $3 \mathrm{~mm}$. Ce profil de vitesse permet de déterminer les valeurs de skewness $S k$, caractérisant une dissymétrie en vitesse, ainsi que d'asymétrie $A s$, qui caractérisent une dissymétrie d'accélération à toutes les altitudes. Le Skewness et l'Asymétrie sont calculés comme les moments d'ordre 3 de la vitesse et de l'accélération respectivement. 
Alors que l'asymétrie diminue au fur et à mesure que l'on se rapproche du fond, le skewness augmente avant de baisser à nouveau. Nous voulons voir si ce comportement est reproduit par un modèle numérique $1 \mathrm{D}$ de type $k$ - $\omega$, décrit ci-après.
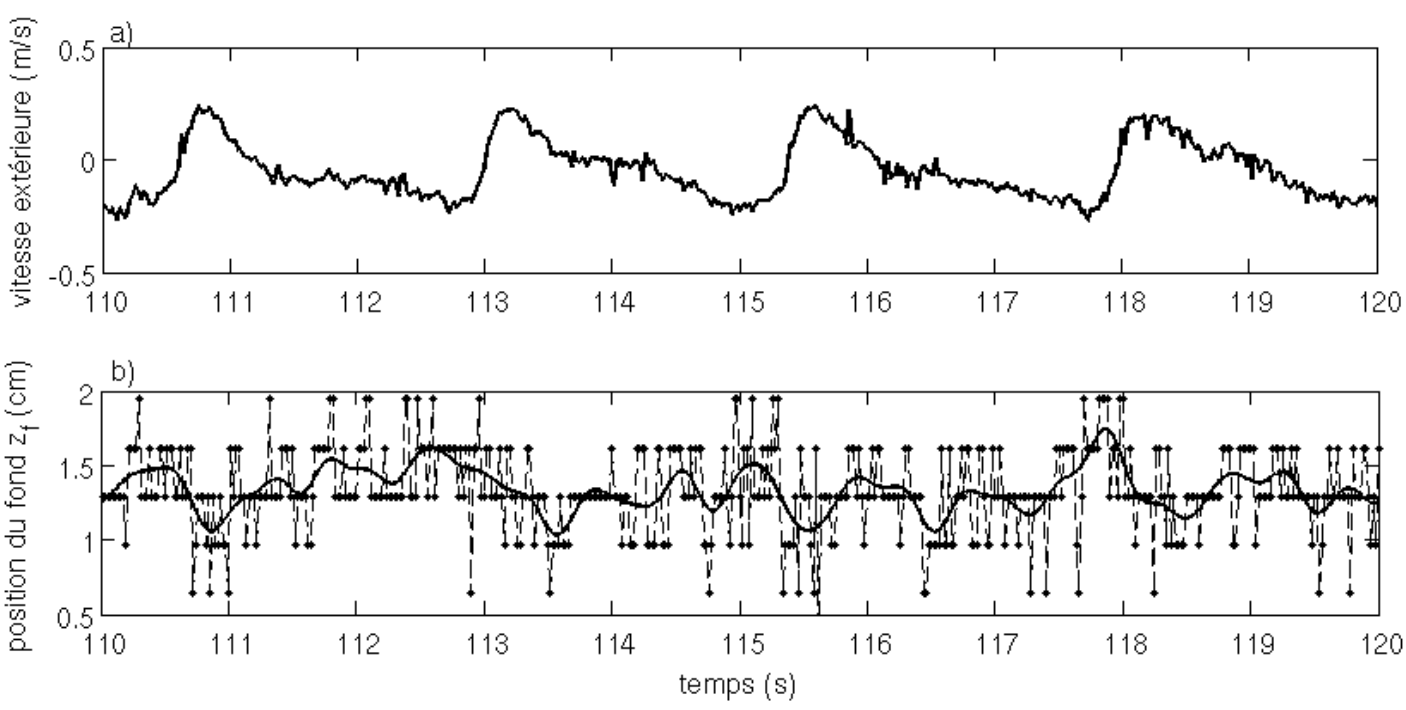

Figure 3. a) vitesse u à l'extérieur de la couche limite, pendant 10 secondes.

b) positions du fond mesurées (traits tiretés) et filtrées (trait plein).

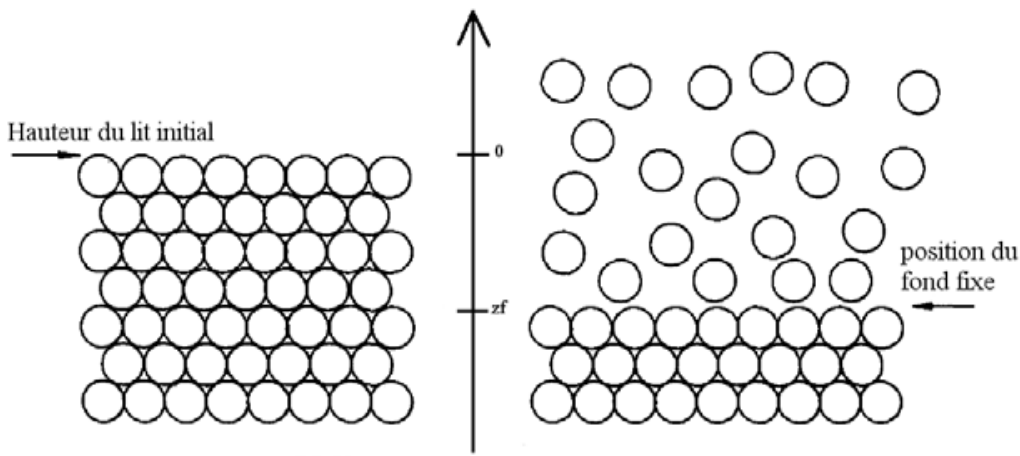

Figure 4. Définition du lit initial et de la position du lit fixe d'après DICK \& SLEATH (1991). La position $z=0$ correspond à la hauteur du lit initial.

\subsection{Modèle numérique}

Pour la partie numérique nous utiliserons une modèle de couche limite turbulente $1 \mathrm{D}$ de type $k$ - $\omega$ (WILCOX 2006 ; GUIZIEN et al., 2003) dans sa version à bas nombre de Reynolds. La vitesse horizontale dans la couche limite $u$, l'énergie cinétique turbulente $k$ et le taux de dissipation d'énergie $\omega$ sont solutions des équations (1-3) :

$\frac{\partial u}{\partial t}=-\frac{1}{\rho} \frac{\partial P}{\partial \cdot r}+\frac{\partial L^{-}}{\partial t}+\frac{\partial}{\partial z}\left(\left(\nu+\nu_{l}\right) \frac{\partial u}{\partial z}\right)$ 


$$
\begin{aligned}
& \frac{\partial k}{\partial t}=\nu_{l}\left(\frac{\partial u}{\partial z}\right)^{2}-j k u+\frac{\partial}{\partial z}\left(\left(\nu+\sigma_{k} \nu_{l}\right) \frac{\partial k}{\partial z}\right) \\
& \frac{\partial u}{\partial t}=n \nu_{1} \frac{u}{k}\left(\frac{\partial u}{\partial z}\right)^{2}-k^{2}+\frac{\partial}{\partial z}\left(\left(\nu+\sigma_{\mathrm{ct}} \nu_{l}\right) \frac{\partial u}{\partial z}\right)
\end{aligned}
$$

Les coefficients et conditions aux limites à utiliser sont explicités dans (GUIZIEN et al., 2003). La série temporelle de vitesse extérieure $u_{\infty}(t)$ utilisée comme condition à la limite pour le modèle numérique, est la série de vitesse obtenue expérimentalement à la hauteur pour laquelle on observe le maximum de la vitesse Urms, qui correspond à $z=3,6 \mathrm{~cm}$. Cette vitesse est celle utilisée dans les 23 séquences, mises bout à bout, et on itère le modèle numérique jusqu'à obtenir une convergence suffisante.

Concernant la rugosité équivalente $k_{s}$ utilisée dans le modèle, celle ci est prise égale à

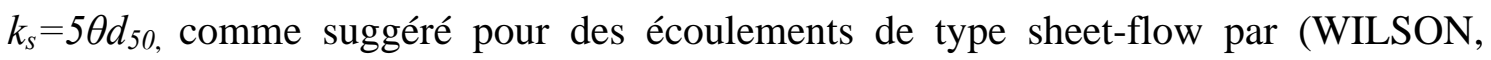
1989 ; DICK \& SLEATH, 1991), où $\theta$ représente le nombre de Shields, et $d_{50}$ le diamètre médian du sédiment.

\subsection{Caractéristiques des évolutions du lit fixe}

Grâce aux données fournies par l'ADVP, nous pouvons déterminer la position $z_{f}(t)$ du niveau du fond fixe (BERNI, 2011). La résolution verticale de l'ordre de $3 \mathrm{~mm}$ s'observe sur la figure 5. Néanmoins, la position aléatoire du fond $z_{f}$ peut être modélisée par une fonction de densité de probabilité, dont les paramètres statistiques sont ceux déterminés empiriquement. Nous pouvons voir sur la figure 5, une distribution normale obtenue avec la déviation standard de la position du fond, ainsi qu'une distribution de Pearson de type IV ayant les mêmes moments d'ordre 2, 3 et 4 que la distribution.

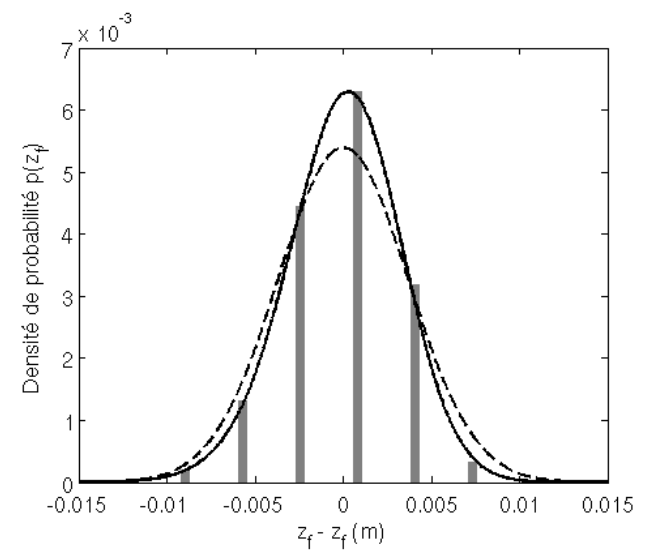

Figure 5. fonctions de densité de probabilité p(zf) équivalente à la distribution des positions du fond, qui correspondent à une distribution normale (traits tiretés) et à une distribution de type Pearson (trait plein) avec les mêmes paramètres statistiques.

L'histogramme représente la distribution des données expérimentales. 


\section{Résultats}

\subsection{Modèle numérique sur fond fixe}

Les résultats obtenus avec le modèle numérique $k$ - $\omega$ sur fond fixe (figure 6) montrent que le modèle numérique reproduit le comportement observé lors de l'expérience, c'est à dire une augmentation de la skewness près du fond, ainsi qu'une diminution de l'asymétrie, mais le pic ne se situe pas à la même hauteur. En effet, en définissant $\delta=(2 v / \omega)^{0,5}$ qui correspond à la longueur de Stokes, le modèle numérique sur fond fixe nous donne un maximum de skewness pour $z=2 \delta$, contre un maximum situé à $z=25 \delta$ pour l'expérience, et la vitesse Urms prédit une vitesse maximale pour $z=3.5 \delta$, alors que les données expérimentales situent le pic à $z=35 \delta$. Il semble que le modèle numérique est capable de reproduire les phénomènes non-linéaires observés dans la couche limite, mais la diffusivité verticale n'est pas suffisante. Si on regarde les résultats obtenus avec une rugosité 10 fois supérieure aux valeurs théoriques pour des couches limites oscillantes sur fond mobile, on observe bien sur la figure 6 une diffusion verticale des valeurs moyennes de Urms, $A s$ et $S k$, mais pas suffisantes pour pouvoir expliquer la diffusivité verticale des valeurs expérimentales.

\subsection{Effets de fond fixe variable}

Nous voulons donc vérifier si en couplant le modèle numérique, implémenté pour un fond fixe, avec les positions du fond $z_{f}$, il est possible d'expliquer en partie cette diffusion verticale observée expérimentalement.

Nous disposons de manière synchronisée d'une série temporelle de vitesse $u_{\infty}(t)$ et de position du lit $z_{f}(t)$. On construit alors à l'aide du modèle numérique $k$ - $\omega$ une série synchronisée de vitesse de $u(z, t)$ pour toute altitude dans la couche limite. La moyenne d'ensemble sera alors :

$U(z)=\frac{1}{N} \sum_{j=1}^{N} u\left(z-z_{f}(j)\right)$

$N$ étant le nombre total de points de la série de $u_{\infty}(t)$.

Comme nous pouvons le voir dans la figure 6 , ce couplage nous donne des profils de vitesse, d'asymétrie et de skewness en escaliers, du fait de la résolution de l'ADVP qui ne nous permet pas de connaître la position du fond avec plus de précision. On remarque tout de même qu'aux hauteurs correspondantes aux points de mesures, les valeurs du modèle sont proches des valeurs expérimentales pour la vitesse, ainsi que pour le skewness pour des hauteurs inférieures à $20 \delta$.

De façon plus approximative et décorrélée dans le temps, nous pouvons approcher les profils verticaux déterminées expérimentalement par moyenne d'ensemble, comme la convolution du $U r m s_{0}(z), S k_{0}(z)$ et $A s_{0}(z)$ (profils verticaux sur fond fixe) par la densité de probabilité $p\left(z_{f}\right)$ des positions du fond, ainsi : 
$\operatorname{Urms}(z)=\int_{-\infty}^{\infty} p\left(z_{f}\right) U r m s_{0}\left(z-z_{f}\right) d f z_{j}$

Cette formulation est semblable au couplage avec la position du fond, avec la différence que les positions du lit et les profils de vitesses correspondants ne sont plus synchronisés dans le temps. Une formule similaire est mise en œuvre pour estimer $A s(z)$ et $S k(z)$.

Les profils obtenus par convolution (figure 6) donnent des résultats proches des valeurs expérimentales, et ont des pentes semblables aux données expérimentales lorsque l'on se trouve près du fond, pour $z<20 \delta$. Ces résultats semblent indiquer que la diffusion verticale au niveau des profils moyens de vitesse, d'asymétrie et de skewness peut en partie s'expliquer par la mobilité du fond, qui agit comme un diffuseur pour les valeurs moyennes. Cependant, certaines caractéristiques des valeurs moyennes échappent à cette approche. En particulier le pic expérimental de skewness en $\mathrm{z}=25 \delta$.
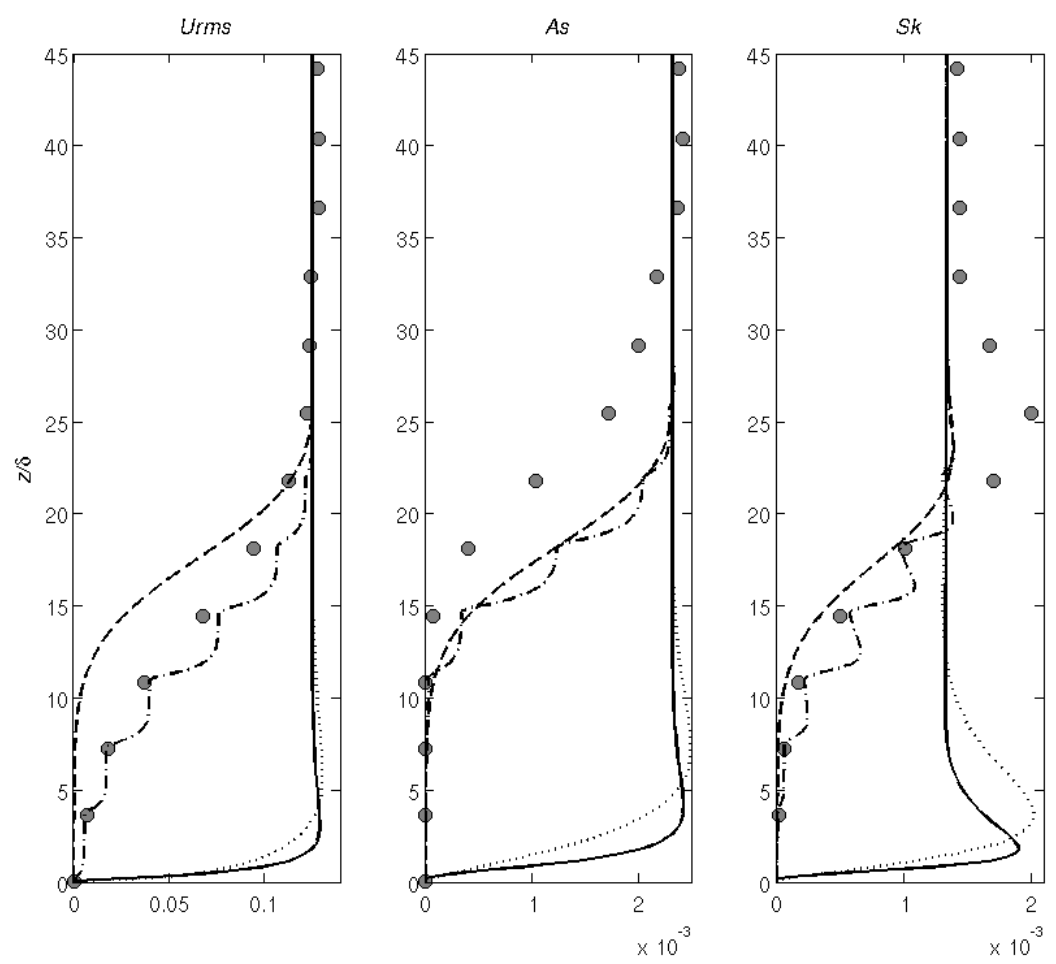

Figure 6. Profils moyens de vitesse Urms, d'asymétrie As et de skewness Sk sur l'ensemble des réalisations. (•) représente les profils obtenus expérimentalement par moyenne d'ensemble à chaque altitude, (-) les profils Urms0, Sk0 et As0 du modèle numérique obtenus en considérant une rugosité de fond égale à 10 fois la rugosité théorique,(--) les profils obtenus par convolution décorrélée avec une distribution de type Pearson, (-.-.) les profils obtenus par couplage entre la position du fond et les profils de vitesses sur fond fixe. 


\section{Conclusions}

Un post-traitement combinant des résultats d'un modèle numérique $1 \mathrm{D}$ de type $k$ - $\omega$ avec une série temporelle de positions du niveau du lit fixe permet d'obtenir des profils d'asymétrie et de skewness semblables à ceux obtenus avec les données expérimentales, et permettent en partie d'expliquer une diffusivité verticale de ces paramètres sur fond mobile.

L'aspect intéressant de la validation du modèle, est que les caractéristiques non linéaires au sein de la couche limite se retrouvent au niveau des ordres de grandeur. Il faudrait plus en avant étudier l'évolution des non-linéarités au sein de la couche limite, qui sont importantes dans l'estimation de la contrainte de cisaillement et de son déphasage par rapport à la vitesse extérieure à la couche limite.

\section{Remerciements}

Le premier auteur remercie CONICYT de lui avoir accordé une bourse de doctorat, la Pontificia Universidad Católica, ainsi que le laboratoire LEGI pour son accueil.

\section{Références bibliographiques}

BERNI C. (2011). Processus de mobilisation et de transport de sédiments dans la zone de déferlement, Thèse de l'Université de Grenoble, 231 p.

DICK J.E., SLEATH J.F.A. (1991). Velocities and concentrations in oscillatory flow over beds of sediment. Journal of Fluid Mechanics, Vol. 233, pp 165-196. doi:10.1017/S0022112091000447

GUIZIEN K., DOHMEN-JANSSEN C.M., VITTORI G. (2003). 1DV bottom boundary layer modelling under combined wave and current: suspension ejection events and phase lag effects. J. Geophys. Res., 108(C1): 3016. doi:10.1029/2001JC001292

WILCOX D.C. (2006). Turbulence Modeling for CFD, $3^{\text {rd }}$ edition. DCW Industries, Inc., La Canada CA

WILSON K.C. (1989). Mobile-bed friction at high shear stress, Journal of Hydraulic Engineering, Vol 115, No 6, pp 825-830. doi:10.1061/(ASCE)0733-9429(1989)115:6(825) 